\title{
APPLICATION OF SHAPE MEMORY ALLOY IN HARVESTO-ABSORBER SYSTEM
}

\author{
Krzysztof KĘCIK* \\ *Faculty of Mechanical Engineering, Department of Applied Mechanics, Lublin University of Technology, 20-618 Lublin, Poland \\ k.kecik@pollub.pl \\ received 4 May 2015, revised 27 October 2015, accepted 28 October 2015

\begin{abstract}
This paper presents a conception of the harvester-absorber system consisting of two parts. The first is the pendulum attached to the main system (oscillator), which is suspended on the linear damper and the nonlinear spring made of shape memory alloy. The spring is modelled as a polynomial function based on Landau-Ginzburg theory of phase transitions (similar as ferroelectric and ferromagnets). The obtained results show, that SMA element can increase harvesting energy level, while the absorber effect can be impaired (but not loss). Additionally, introducing SMA element causes changes in dynamics, introduces a new unstable solutions and bifurcations. The analysis was done by classical integration and continuation solution methods.
\end{abstract}

Key words: Pendulum, SMA Spring, Energy Harvester, Vibration Absorber, Control, Continuation Method

\section{INTRODUCTION}

The dynamic vibration absorber is proved to be a very simple and effective vibration suppression device, with many practical implementations in civil and mechanical applications. While, the energy harvesting is a promise to recovery energy from unwanted vibrations (Kecik and Borowiec, 2013). The pendulum mechanism can be used in different mechanical systems for various purposes. The application for vibration absorption or energy harvesting is one of the most common. The dynamic vibration absorbers (DVAs), also called Vibration Neutralizers (VNs) or Tuned Vibration Absorbers (TVAs), are mechanical appendages comprising inertia, stiffness, and damping elements which, once connected to another mechanical system, or structure called the primary system or machine are capable of absorbing on controlling the vibrations (Liao et al., 2011).

In the literature studies (Kaynia et al., 1981; Regis, 2010, Sladek and Klingner, 1983; Soto-Brito and Ruzi, 1999) it is shown, that the effectiveness of linear vibration absorbers is limited to the close neighborhood of a vibration mode. This frequency robustness limitation called for the development of nonlinear vibrations absorber (NLVA). On the most popular NLVA are the autoparametric vibration absorbers (AVAs) (Kecik, 2015). The idea of such solutions lies in attaching the absorber to the primary structure in such a manner that it experiences a parametric base excitation, and therefore, the absorber frequency is tuned around one-half of the troublesome frequency value. The equations of motion of such system include the quadratic nonlinearities, and inertial terms, which can influence parametric resonance (Warminski and Kecik, 2009).

Moreover, such systems exhibit different nonlinear mechanism (e.g., saturation (Oueini et al., 1997), quenching, sub/ superharmonic resonances, autoparametric resonances (Warminski and Kecik, 2009), 0:1 resonances (Lacarbonara, 2012)) can be leveraged to produce effective actuator actions for resonance rejection in nonlinear lumped and distributed-parameter systems. Additionally, they are very sensitive to system's parameter, therefore the control method to maintain demand solution should be applied.

In this aim, the application of smart material (SM) seems very promising. Nowadays, the most used materials are the shape memory alloys (SMA), the piezoelectric materials (PM), the magnetostrictive materials (MSM) and the electro- and magneto-rheological fluids (MRF) (Janocha, 2007). These materials have the ability of changing their shape, stiffness, among other properties, through the imposition of temperature or stress, electrical or electro-magnetic fields. The SMAs are a kind of smart materials whose physical properties change as a function of temperature. This effect can be exploited to build tunable and adaptive devices.

On the other hand, in nonlinear vibrating systems energy harvesting (EH) recovery is possible, too (Kecik and Borowiec, 2013; $\mathrm{Xu}$ and al., 2007; Wiercigroch, 2011). EH has been an active research area and systems refer to devices that capture and transform energy into electricity. Usually, the energy could be recovered from the kinetic energy of moving or vibrating structures. The vibration based energy harvesters can be categorized into three main types, namely: electromagnetic, piezoelectric, and electrostatic (depending on the medium of the transducer).

The civil structures and pendulum absorbers usually exhibit low frequency structural vibrations, which make it difficult for an energy harvester to extract energy. Therefore, tuning of such systems is very important. The vibrational energy harvesters achieve their highest output power nearly resonance regions (because of the highest amplitude of vibration) (Gu and Livermore, 2010). The application of smart elements can increase the resonance region (Kecik, 2015) and improved power recovery. The novel conception for simultaneous vibration absorption and the energy recovery in this paper is proposed. The harvesto-absorber system consists of oscillator with added absorber (pendulum). In the pivot of absorber, the energy harvesting device is mounted.

The proposed conception can be used for swinging or rotation of the pendulum. To control of dynamics, the SMA spring mounted in the suspension of the main system is applied. The SMA spring 
beahavior is described by the polynomial model, which characterizes relationship between strain, stress and temperature. The main idea of this work is influence analysis of nonlinearity of SMA spring on dynamics, absorption effect and energy harvesting problem.

\section{ENERGY HARVESTING DYNAMIC VIBRATION ABSORBER (EHDVA)}

\subsection{Model of a pendulum-like system}

A schematic diagram of the proposed energy harvesting dynamic vibration absorber (EHDVA) is shown in Fig. 1. The system consits of two main parts. The first is a pendulum (absorber) made up two masses $\left(m_{2}\right.$ and $\left.m_{3}\right)$ and a length denoted as $l$. The second part is called the main system (oscillator) suspended on the linear damper (where $c$ is a viscous damping coefficient) and nonlinear SMA spring. Assumed, that the main system can move only in vertical direction.

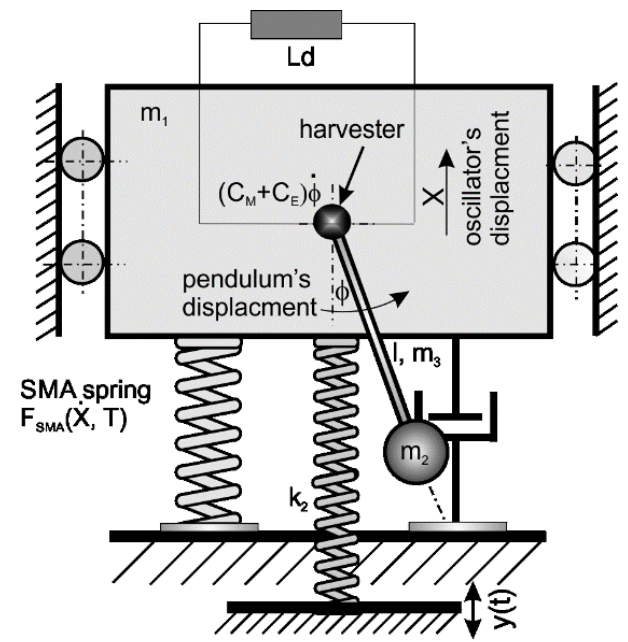

Fig. 1. Schematic diagram of the energy harvesting vibration absorber

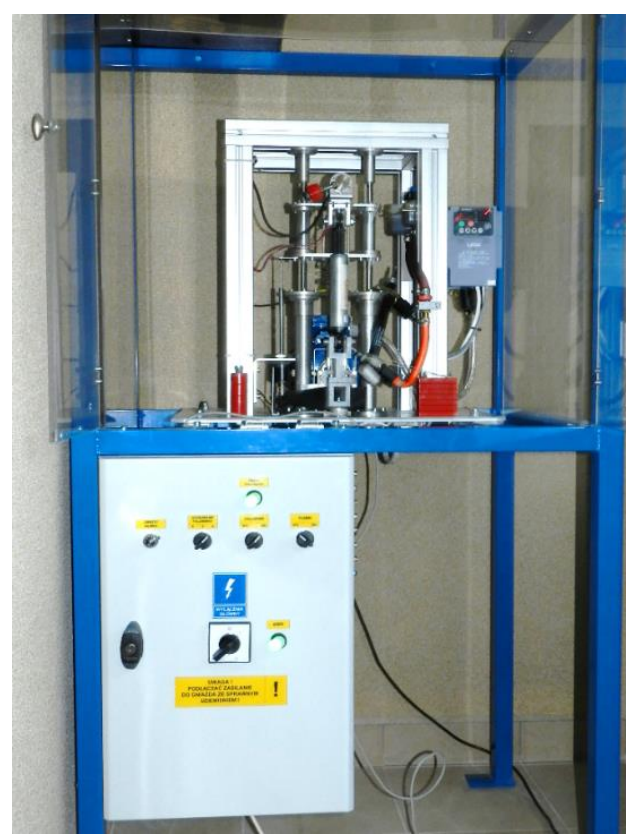

Fig. 2. Real view of EHDVA system with SMA oscillator's spring
The system is excited with help of the linear spring with stiffness denoted as $k_{2}$ (kinetic excitation). The excitation of end spring is a harmonic function $y(t)=Q \cos (\omega t)$. The system has two degree of freedom: $x$ vertical displacement of the oscillator and $\phi$ angular displacement of the pendulum. In the pendulum pivot, the energy harvesting device is mounted. Therefore, the total damping of the pendulum consists of the mechanical viscous $\left(c_{M}\right)$ and electrical $\left(c_{E}\right)$ damping.

The harvester device is connected directly to the electric load $(L d)$. Note that parameters of model identified on the experimental system, presented in Fig. 2. Detailed information about experimental system can be found in Kecik (2015).

\subsection{Model of SMA spring}

The most popular constitutive models for describing the behavior of SMAs come from the original Landau-Ginzburg theory of phase transitions. This theory describes the constitutive information about SMA material by a polynomial free-energy, function whose partial derivatives provide constitutive equations for strain $(\varepsilon)$ and entropy. In Falk (1980), Falk proposed a Landau-Devonshire like free-energy function based on the analogy between SMA uniaxial stress-strain curves and the electric field magnetization curves of ferromagnetic materials. This model assumes a polynomial free energy potential, which allows pseudoelasticity and SME description. The free energy potential $(W)$ is defined as

$W(T, \varepsilon)=\frac{a_{1}}{2}\left(T-T_{1}\right) \varepsilon^{2}-\frac{1}{4} a_{2} \varepsilon^{4}+\frac{a_{2}^{2} \varepsilon^{6}}{24 a_{1}\left(T_{A}-T_{M}\right)^{\prime}}$,

where parameters $a_{1}, a_{2}$ are positive constants, while $T_{M}$ and $T_{A}$ are the temperature below the martensitic and austenite phase are stable, while $T$ is activated SMA temperature.

By applaying the stress-strain relation

$\sigma(\varepsilon, T)=\frac{\partial W(\varepsilon, T)}{\partial \varepsilon}$

and replacing strain by the displacement, obtained function describing ellogation of SMA spring versus temperature

$F_{S M A}(x, T)=\tilde{a}_{1}\left(T-T_{M}\right) x-\tilde{a}_{2} x^{3}+\frac{\tilde{a}_{2}^{2} x^{5}}{4 a_{1}\left(T_{A}-T_{M}\right)}$.

The parameters $\tilde{a}_{1}, \tilde{a}_{2}, \tilde{a}_{3}$ are positive material constants includes cross-section of the SMA material spring.

\subsection{Energy harvesting device conception}

A typical vibration $\mathrm{EH}$ system consists of a mechanical system with external excitation, a transducer that converts the vibration energy into electric energy, mechanisms for motion transmission and magnification, power electronics and energy storage elements, and energy management.

The rotatory harvester device is mounted in the pendulum suspension. The harvester consists of two main elements, a stator and a rotor. Additionally, it includes two identical windings fixed to housing. When the pendulum is rotating or swinging, the converter generates electricity due to magnetic induction. Generally, inductive energy depends on a relative velocity between the coil and the magnet and electrical properties of circuits. Because of small size of harvester device (compared to total system), assumed that it is dynamics not influence on vibrations of other components. The 
scheme of energy harvester system and electric circuit in Fig. 3 is shown. The parameter $\rho$ denotes housing radius, while $\delta$ is a transduction factor, which depends on numbers of coil turns, average magnetic field strength, and the coil length.

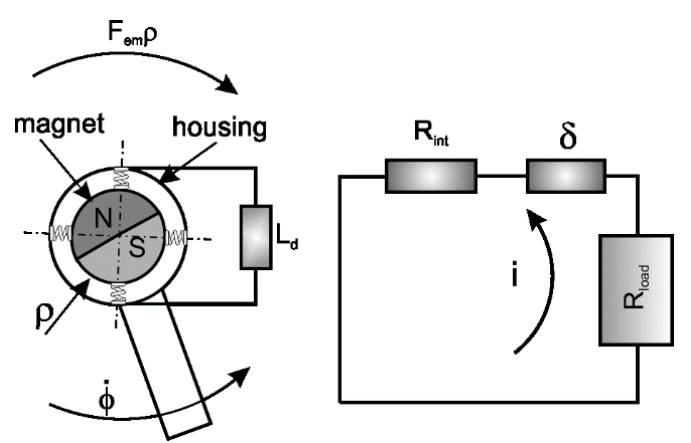

Fig. 3. Schematic diagram of inductive energy and equivalen circuit

The total electromotive (electromagnetic) force generated by the windings denoted $F_{e m}$, can be written as (Ma et al., 2012)

$F_{e m}=\delta i=c_{e} \dot{\varphi}$.

The coupling term of the mechanical system with the electric circuit is directly proportional to the angular velocity and electrical damping of the electrical device (Kecik and Borowiec, 2013). This means that is equivalent to a viscous damping force with a damping coefficient $c_{e}$. From mathematical point of view, equations of motion for the pendulum harversto-absorbers system are identical as system without harvester device. However, the harvester devices slightly increases damping of the pendulum.

To calculate the electrical damping coefficient $c_{e}$, the equation for the electrical circuit is used by applying Kirchhoff's law

$i\left(R_{\text {load }}+R_{\text {int }}\right)-\delta \rho \dot{\varphi}=0$,

where $R_{\text {load }}$ is the resistance of the external load, $R_{\text {int }}$ is the internal resonance.

The recovered voltage can be calculated using classical Ohm's law

$U=i R_{\text {load }}=\delta \frac{\rho R_{\text {load }} \dot{\varphi}}{\left(R_{\text {load }}+R_{\text {int }}\right)^{\prime}}$,

where $i$ denotes current.

Then, the electrical damping coefficient can be calculated

$c_{E}=\frac{\rho \delta^{2}}{\left(R_{\text {load }}+R_{\text {int }}\right)^{\prime}}$

which depends on the construction of harvester device and property of electrical circuit.

\subsection{Dimensional and non-dimesional equations of motion}

The dynamics of the primary mass $\left(m_{1}\right)$, and the absorber $\left(m_{2}\right.$ and $\left.m_{3}\right)$, can be described by two coupled ordinary differential equations (ODE). The equations are coupled by inertial terms, which is typical for autoparametric systems. The equations of motion of model presented in Fig. 1 have form:

$\left(m_{1}+m_{2}+m_{3}\right) \ddot{x}+c \dot{x}+F_{S M A}(T, x)+\left(m_{2}+m_{3} / 2\right) l$

$\left(\ddot{\varphi} \sin \varphi+\dot{\varphi}^{2} \cos \varphi\right)=k_{2} Q \cos (\omega t)$, $\left(m_{2}+m_{3}\right) l^{2} \ddot{\varphi}+\left(c_{M}\right) \dot{\varphi}+\left(m_{2}+m_{3} / 2\right) l(\ddot{x}+g) \sin \varphi$ $+\delta i=0$,

where $F_{S M A}$ describes force of SMA spring which depends of temperature and its deflection.

Introducing dimensionless time $\tau=\omega_{o} t$, where $\omega_{o}$ is a natural frequency of the oscillator, $X=x / x_{s t}$ (where $x_{s t}$ is a static displacement of the oscillator) and replacing part $\delta i$ (eq. (4)) obtained eqs. (8) and (9) in dimensionless form are:

$\ddot{X}+\alpha_{1} \dot{X}+(\theta-1) X-\beta_{1} X^{3}+\beta_{2} X^{5}+$

$\mu \lambda\left(\ddot{\varphi} \sin \varphi+\dot{\varphi}^{2} \cos \varphi\right)=q \cos (\vartheta \tau)$,

$\ddot{\varphi}+\left(\alpha_{2}+\kappa\right) \dot{\varphi}+\lambda(\ddot{X}+1) \sin \varphi=0$.

The dimesionless parameters are defined:

$\tau=\omega_{0} t, \quad \omega_{0}^{2}=\sqrt{\frac{a_{1} T_{M}}{M}}, \quad X=\frac{x}{x_{s t}}, \quad \vartheta=\frac{\omega}{\omega_{0}}, \quad \theta=\frac{T}{T_{M}}$,

$\theta_{c}=\frac{T_{A}}{T_{M}}, \quad \beta_{1}=\frac{a_{2} x_{s t}^{2}}{\omega_{0}^{2} M}, \quad \beta_{2}=\frac{\beta_{1}^{2}}{4\left(\theta_{c}-1\right)}, x_{s t}=\frac{g M}{a_{1} T_{M}}$,

$M=\sum_{i=1}^{3} m, \alpha_{1}=\frac{c}{\omega_{0} M}, \alpha_{2}=\frac{c_{M}}{\omega_{0} l^{2}\left(m_{2}+m_{3} / 3\right)}$,

$\lambda=\frac{\left(m_{2}+m_{3} / 2\right) x_{s t}}{\left(m_{2}+m_{3} / 3\right) l}, \mu=\frac{\left(m_{2}+m_{3} / 2\right) l^{2}}{M x_{s t}^{2}}$,

$q=\frac{k_{2} Q}{\left(k+k_{2}\right) x_{s t}}, \quad \kappa=\frac{c_{E}}{\left(m_{2}+m_{3} / 3\right) l \omega_{0}}$.

The dimensionless equations of motion are easy to analysing because of lack of units and small number of parameters.

In the dimensionless form the eq. (3) resorting force of SMA spring has a form

$F_{S M A}(X, T)=(\theta-1) X-\beta_{1} X^{3}+\beta_{2} X^{5}$,

where definition of dimesionless material constant are explained in (12). Note, for $\beta_{1}=\beta_{2}=0$ and $\theta=2$, SMA's model exhibits linear behavior like classical linear spring. The examplary characteristics of SMA spring model in Fig. 4 are presented. The black line presents classical linear characteristics, while blue line denotes temperature $\theta=0.75$, green $\theta=1$ and red $\theta=1.5$.



Fig. 4. Force-displacement characteristics of SMA spring for $\beta_{1}=0.6$, $\beta_{2}=0.9$

The proposed model shows strongly nonlinear behaviour, especially for large displacement. For small value of displacement $(x<0.5)$ characteristics are close to linear. All parameters describing the harvesto-absorber system and SMA spring are identified on the laboratory rig. 


\section{NUMERICAL ANALYSIS}

\subsection{Influence of the SMA spring on absorption effect}

The absorption region for the pendulum absorber system with the classical linear spring is known very well (Warminski and Kecik, 2006). This region, existing for properly tuned system, near the resonace peak (Kecik et al., 2014). However, for system with the SMA spring the dynamics can diametrically changed. Considering the system as a dynamic absorber, it is necessary to understand the infuence of SMA stiffness spring on this effect. Additionally, the application of SMA spring in oscillator's suspension can be an alternative way to control the absorber dynamics. The suspension with the SMA spring is easy to apply in the real construction. Note, that the control by the element mounted in the pendulum pivot (e.g. rotatory damper) is difficult due to the harvester. Alternative control for such systems can be realized by the oscillator's damper. In papers (Cartmell and Lawson, 1994; Vazquez-Gonzalezal and SilvaNavarro, 2008), the effective control by magnetorheological damper mounted in the oscillator's suspension is proposed.



Fig. 5. Frequency response curves for the oscillator, for $\beta_{1}=0.6, \beta_{2}=0.9$ and different temperature ratio

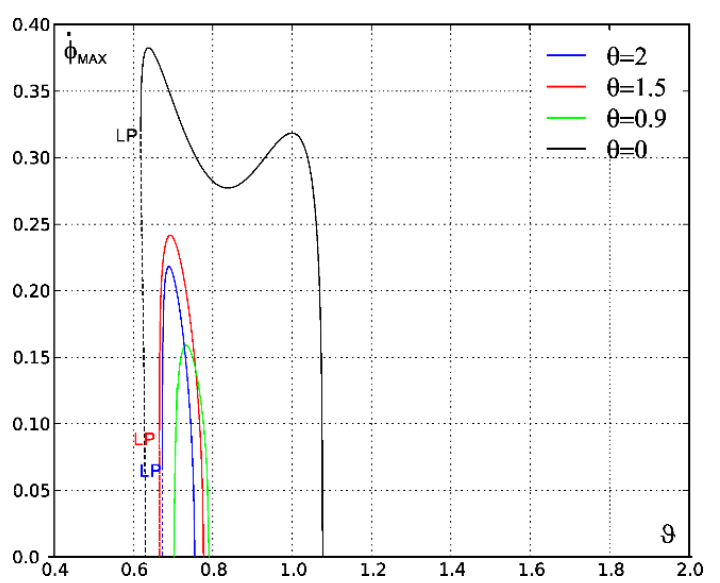

Fig. 6. Frequency response curves for the pendulum velocity, for $\beta_{1}=0.6$, $\beta_{2}=0.9$ and different temperature ratio

The calculations in this section have been performed using software for numerical continuation, Auto07p (Doedel at al., 2011). The results that have been obtained by continuation give us an overview of system dynamics demonstrating all possible periodic solutions. The exemplary frequency response curves are presented in Fig. 5.

The simulation parameters are: $\alpha_{1}=0.32, \alpha_{2}=0.0769, q=0.9$, $\mu=17.2, \lambda=0.12, \kappa=0.0031$. The dashed dotted line denotes unstable, while continous lines stable solutions. The labeled point describes the bifurcations points (PD -pediod doubling, LP - limit (fold) point, BP branch point).

The frequency response curves, for harvesto-absorber system with clasical spring $\left(\theta=2, \beta_{1}=\beta_{2}=0\right)$, marked by the black line in Figs. 5 and 6 are presented. The absorption region is clearly visible, for frequency range $\vartheta \approx 0.6-1.05$. Unfortunatelly, for system with the SMA spring, the absorption region is very small, and located between 0.65 and 0.8 . The size of this region depends on the temperature, and additionaly, the effectivness level of vibration absorption is very low. However, the amplitude of semi-trivial solution (vibrates only the main system) is significantly reduced. The resonance curves were bent in the rigt side, and for lower temperature, the semi trivial solution changes in unstable (see, the green line in Fig. 5).

The SMA spring can be used to control of the pendulum velocity, as shown in Fig. 6. The temperature changes cause increase or decrease in the pendulum velocity. This effect can be used to find compromise beetween vibration absorption and energy harvesting. Note, that application of SMA spring reduces non-trivial solution from right side (from the higher frequency).

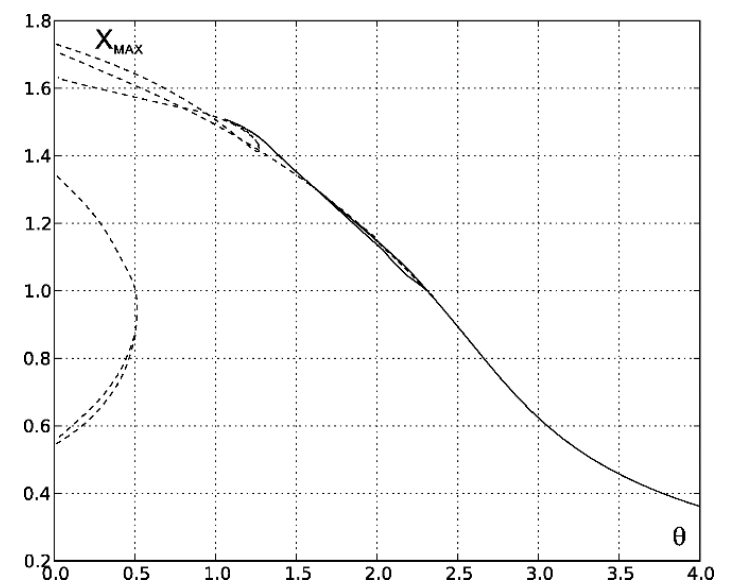

Fig. 7. Influence of temparature on amplitude of the oscillator, for $\vartheta=0.7$ (absorption region), $\beta_{1}=0.6, \beta_{2}=0.9$

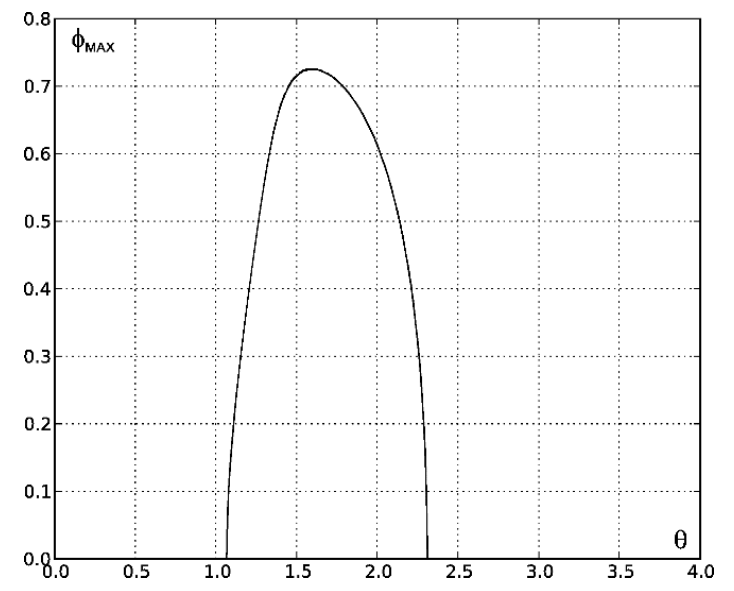

Fig. 8. Influence of temparature on amplitude of the pendulum, for $\vartheta=0.7$ (absorption region), $\beta_{1}=0.6, \beta_{2}=0.9$ 
The effect of temperature (parameter $\theta$ ) on the oscillator and pendulum amplitudes has been investigated (Figs. 7 and 8). The stable solutions are observed for $\theta>1$. The pendulum executes motions for range of $\theta$ from 1.1 to 2.3 .

\subsection{Influence of SMA spring on energy harvesting}

The energy harvesting level strongly depends on the construction of the harvester device. Usually, these devices are mounted in the pendulum suspension. This construction is very difficult to control by applied the pendulum's damping. Therefore, this paper proposes allternative method of control by SMA element mounted in the suspension. In this section influence of SMA spring on energy harvesting is studied in detail.

Firstly, the influence of temperature on recovery voltage is analysed (Fig. 9). The resonance region is clearly visible, nerly frequency $\vartheta=0.7-0.8$. This result agrees with the resonance curves, presented in Figs. 7 and 8 . The maximal recovered voltage was about $6 \mathrm{mV}$. Of course, the values depend on electrical parameters of harvester (for analysis taken: $R_{\text {int }}=55 \Omega, \quad R_{\text {load }}=140 \Omega$, $\delta=7.752 \mathrm{Vs} / \mathrm{m}, \rho=0.01 \mathrm{~m})$. The range of temperature in which voltage is recovered equals $\theta=0.5$ to $\theta=2.3$, but the best is located for $\theta=1.5$ to $\theta=2$.

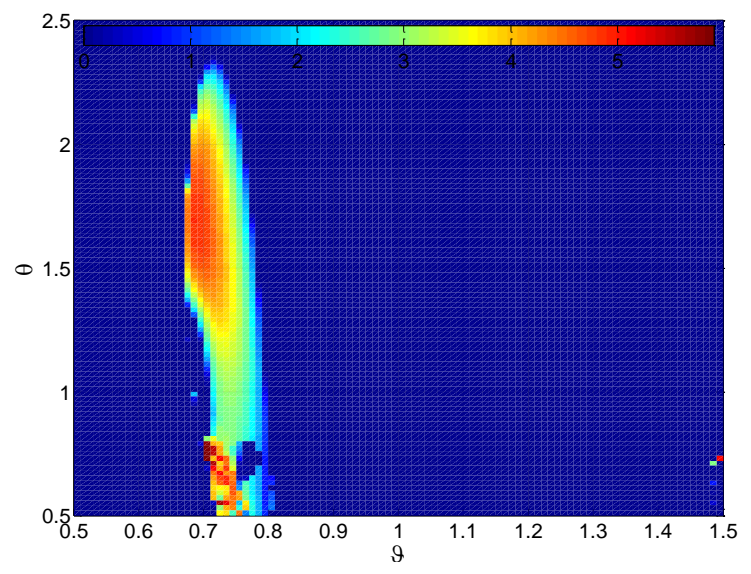

Fig. 9. The 3D plot: temperature vs. frequency vs. voltage, for $\beta_{1}=0.6, \beta_{2}=0.9$

Next, parameters describing material of which the SMA spring is made $\left(\beta_{1}, \beta_{2}\right)$ are analyzed. The parameter $\beta_{1}$ depends of natural frequency of the oscillator and static displacement, also (see eq. (12)). While, parameter $\beta_{2}$ comes from $\beta_{1}$ and depends on austenite and martesite temperature phases.

The relationship beetwen temperature $\theta$ and parameter $\beta 1$ versus voltage harvesting in Fig. 10 is shown as 3D plot. This result was obtained near the absorption region (for frequency $\vartheta=0.7$ ). The maximal recoverd voltage equals $6 \mathrm{mV}$, and can be recoverd for $\theta=1.4-2.4$ in range of $\beta_{1}$ larger then 0.4 . Additionally, the small regions in which $6 \mathrm{mV}$ recovered can be observed for lower temperature (martensite phases temperature). However, these regions are narrow.

The second parameter $\beta_{2}$ is much more difficult to choose. The region with high level of energy recovery $(12 \mathrm{mV})$ is very narrow (Fig. 11). Moreover, this parameter depends on $\beta_{1}$ (eq. (12)). The obtained results from both diagrams show that properly choosen parameters can incrase recovered voltage.

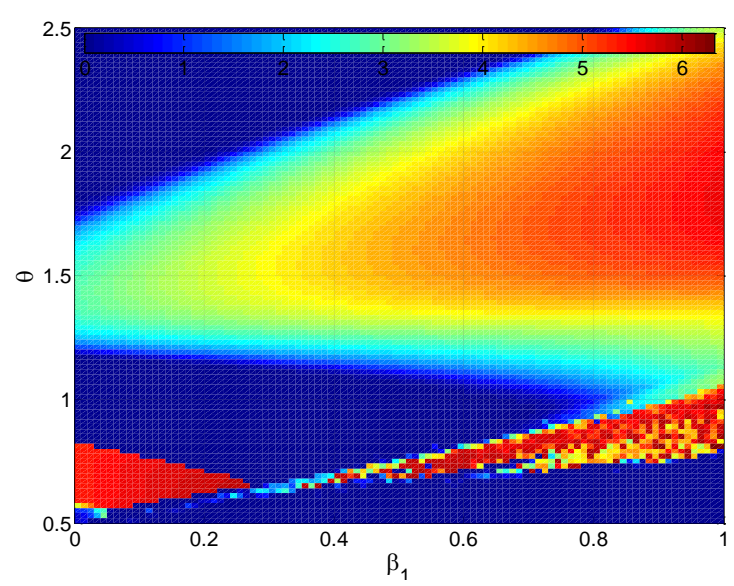

Fig. 10. The 3D plot: temperature vs. parameter $\beta_{1}$ vs. voltage, for $\beta_{2}=0.9$

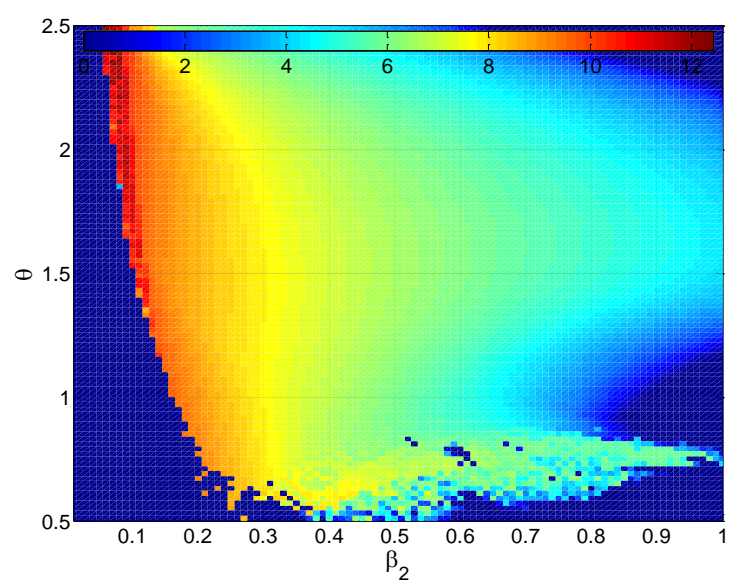

Fig. 11. The 3D plot: temperature vs. parameter $\beta_{2}$ vs. voltage, for $\beta_{1}=0.6, \vartheta=0.7$

The influences of total damping (mechanical and electrical) on energy harvesting is shown in Fig. 12. The critical damping has practically similar value, equals about $0.07-0.08$. Note, that for the pendulum damping close to zero and temperature $\theta=0.5-0.9$, the recovered energy is highest and equals about $12 \mathrm{mV}$.



Fig. 12. The 3D plot: temperature vs. parameter $\beta_{2}$ vs. voltage, for $\beta_{1}=0.6, \vartheta=0.7$

All the above results presented in this section have been done for fixed initial conditions: $X=\dot{X}=\dot{\varphi}=0$ and $\varphi=0.1$. 
The obtained results show, that properly confiruration of SMA parameters $\left(\vartheta, \beta_{1}\right.$ and $\left.\beta_{2}\right)$ can increase energy recovery. However, the application of SMA spring in dynamic absorbers should be detailed checked by numerical studies.

\section{CONCLUSIONS AND FINAL REMARKS}

This paper presents an autoparametric system with added harvester device mounted in the pendulum pivot. This conception of rotatory pendulum harvester, practically prevents control of motion by the pendulum's damping. Therefore, the other solution based on SMA spring mounted in suspension is proposed.

The conception was analyzed in two aspects. Firstly the influenece of SMA spring on absorption region was studied. Next, the problem of energy harvesting under SMA spring was analyzed. The SMA spring significantly reduce the absorption effect (but not eliminate it), what is problem for dynamic absorbers. The frequency response curves for SMA spring are bents in the right side. For, some parameters the solution changes from stable into unstable motion and a new bifurcation point can appears.

The SMA spring significantly influence on energy recovery. Change in the SMA springs parameters the increase or decrease enegy harvesting is possible. The best range of the temperature for $\mathrm{EH}$ equals $\theta=1.5-2$. The properly choose of SMA parameters can incraeases recovered voltage up to $12 \mathrm{mV}$.

The next step will be experimental verification of obtained results and appied control algorithm to highest energy recovery.

\section{REFERENCES}

1. Cartmell M. P., Lawson J. (1994), Performance enhancement of an autoparametric vibration absorber by means of computer control, Journal of Sound and Vibration, 17(2), 173-195.

2. Doedel E., Oldeman B., Champneys A., Dercole F., Fairgrieve, Kuznetsov Y., Paenroth R., Sandstede B., Wang X., Zhang C. (2011), AUTO-07P: Continuation and bifurcation software for ordinary differential equations, Concordia University (Montreal, Canada, 2011).

3. Falk F. (1980), Model free energy mechanics and thermodynamics of shape memory alloys, Acta Metallurgica, 28, 1773-1780.

4. Gu L., Livermore C. 2010), Pendulum-Driven Passive Self-Tuning Energy Harvester for Rotating Applications, Presented at Power MEMS Workshop, Leuven, Belgium.

5. Janocha H. (Ed.), (2007), Adaptronics and Smart Structures, Springer-Verlag, Berlin Heidelberg New York.

6. Kaynia A. M., Veneziano D., Biggs J. M. (1981), Seismic effectiveness of tuned mass dampers, Journal of Structural Engineering, 107(8), 1465-1484.
7. Kecik K. (2015), Dynamics and control of an autoparametric system, International Journal of Non-linear Mechanics, 70, 63-72.

8. Kecik K., Borowiec M. (2013), An autoparametric energy harvester, Th Europen Physical Journal Special Topics, 222(7), 1597-1605.

9. Kecik K., Mitura A., Sado D., Warminski J. (2014), Magnetorheological damping and semi-active control of an autoparametric vibration absorber, Meccanica, 49(8), 1887-1900.

10. Lacarbonara W. (2012), Nonlinear dynamics enabled systems design and control, Journal of Physics: Conference Series, 382, ID 012001, $1-9$.

11. Liao G. J., Gong X. L., Kang C. J., Xuan S. H. (2011), The design of an active-adaptive tuned vibration absorber based on magnetorheological elastomer and its vibration attenuation performance, Smart Material Structure, 20, ID 075015, 1-11.

12. Ma T. W., Zhang H., Xu N. S. 2012), A novel parametrically excited non-linear energy harvester, Mechanical Systems and Signal Processing, 28, 323-332.

13. Oueini S. S., Nayfeh A. H., Golnaraghi M. F. A. (1997), A theoretical and experimental implementation of a control method based on saturation, Nonlinear Dynamics, 13, 189-202.

14. Regis V. (2010), Tuning Methodology of Nonlinear Vibration Absorbers Coupled to Nonlinear Mechanical Systems, PhD Thesis.

15. Sladek J. R., Klingner R. E. (1983), Effect of tuned mass dampers on seismic response, Journal of the Structural Division, 109, 2004-2009.

16. Soto-Brito R., Ruiz S. E. (1999), Influence of ground motion intensity on the effectiveness of tuned mass dampers, Earthquake Engineering and Structural Dynamics, 28, 1255-1271.

17. Vazquez-Gonzalezal B, Silva-Navarro G. (2008), Evaluation of the autoparametric pendulum vibration absorber for a Duffing system, Shock and Vibration, 15, 355-368.

18. Warminski J., Kecik K. (2006), Autoparametric vibrations of a nonlinear system with pendulum. Mathematical Problems in Engineering, 1-19.

19. Warminski J., Kecik K. (2009), Instabilities in the main parametric resonance area of mechanical system with a pendulum, Journal of Sound Vibration, 332, 612-628.

20. Wiercigroch M., Najdecka A., Vaziri V. (2011), Nonlinear Dynamics of Pendulums System for Energy Harvestin, Vibration Problems ICOVP 2011, Book Series: Springer Proceedings in Physics, Edited by: Naprstek J., Horacek J., Okrouhlik M., Marvalova B., Verhulst F., Sawicki J.T., 139, 35-42.

21. Xu X., Pavlovskaia E. E., Wiercigroch M., Romeo F., Lenci S. (2007), Dynamic interactions between parametric pendulum and electro-dynamical shaker, ZAMM Journal of applied mathematics and mechanics, Zeitschrift für angewandte Mathematik und Mechanik, 87(2), 172-186.

Acknowledgments: This work was financially supported under the project of National Science Centre according to decision no. DEC2013/11/D/ST8/03311. 